\title{
Epidemiology of sharp injuries - Prospective EPINet data from five tertiary care hospitals in India - Data for 144 cumulated months, 1.5 million inpatient days
}

\author{
Murali Chakravarthy ${ }^{a, *}$, Sanjeev Singh ${ }^{c}$, Anita Arora ${ }^{d}$, \\ Sharmila Sengupta ${ }^{e}$, Nita Munshi ${ }^{f}$, Sukanya Rangaswamy ${ }^{b}$, \\ Ravneet Kaur ${ }^{e}$, Sohini Arora ${ }^{f}$, Leema Pushparaj ${ }^{b}$, Fini Joseph ${ }^{c}$, \\ Anupama Nair ${ }^{c}$, Daisy Mathew ${ }^{d}$, Zipporah Meritta ${ }^{b}$, Janu Rajagopal ${ }^{c}$, \\ Neelakshi Kumari ${ }^{f}$, Asmita Kamble \\ ${ }^{a}$ Chief Consultant Anesthesiologist, Chairman, Central Hospital Infection Prevention and Control Committee, \\ Fortis Hospitals, Bangalore, Karnataka 560076, India \\ ${ }^{\mathrm{b}}$ Fortis Hospitals, Bangalore, Karnataka 560076, India \\ ${ }^{\mathrm{c}}$ Amrita Institute of Medical Sciences, Cochin, India \\ ${ }^{\mathrm{d}}$ Fortis Escorts Heart Institute, New Delhi, India \\ ${ }^{\mathrm{e}}$ Fortis Hospitals, Noida, India \\ ${ }^{\mathrm{f}}$ Ruby Hall Clinic, Pune, India
}

\section{A R T I C L E I N F O}

Article history:

Received 7 June 2013

Accepted 25 September 2013

Available online 11 October 2013

Keywords:

Sharp injury

Safety products

Healthcare workers

Needle stick

Indian hospitals

\section{A B S T R A C T}

Aims: Sharp injuries (SIs) jeopardize the safety of healthcare workers and are avoidable. This prospective multi-centric study aimed at understanding the epidemiology of sharp injuries in India. This study comes as a sequel to our earlier study, conducted at the same hospitals.

Methods: The cumulated prospective data of 144 months collected from five participating hospitals during the years 2008-2013 and analysed using the EPINet format.

Results: During the study period of 2008-2013, there were more than 1.5 million inpatient (IP) days, and 666 SIs (0.43 SIs/1000 IP days) were observed. The data from June 2008 through May 2009 was named data A and from June 2009 through June 2011 as data B. The data in its entirety and the comparison of data A and B was made. The cumulated data showed that the SIs in the patient's room decreased from 0.9 to 0.2 SIs/1000 IP days $(27.8 \%$ $-14.3 \%$ ). This observation was made in the data from all the participating hospitals; possibly due to providing sharps disposal cans by patient bedside, in addition to aforementioned awareness and educational activities. The SIs among nurses doubled (from 0.01 to $0.02 / 1000$ IP days) and increased among housekeeping personnel.

\footnotetext{
* Corresponding author. Tel.: +91 80 66214444; fax: +91 8066214242.

E-mail address: mailchakravarthy@gmail.com (M. Chakravarthy).

2213-3984/\$ - see front matter Copyright @ 2013, INDIACLEN. Publishing Services by Reed Elsevier India Pvt Ltd. All rights reserved. http://dx.doi.org/10.1016/j.cegh.2013.09.002
} 
Conclusion: SIs caused during the period commencing from completion of injection to disposal decreased in all the participant hospitals, suggesting that the healthcare workers handled the sharps appropriately; however, probably due to wrong disposal, personnel involved in waste disposal got more injuries. Safety engineered devices are not implemented in Indian hospitals despite data supporting their use decreasing SIs.

Copyright @ 2013, INDIACLEN. Publishing Services by Reed Elsevier India Pvt Ltd. All rights reserved.

\section{Introduction}

Sharp injuries (SIs) among healthcare workers appear to be occupational hazard among them. It is prudent to prevent SIs from safety and economic perspective. The pattern of SIs among healthcare workers (HCWs) differs from one facility to another, one society to another and one country to another. ${ }^{1}$ Despite such variations, data from various countries consistently show that nurses are common victims. ${ }^{2-5}$ We published our earlier work, which showed that data from India (Data A), was in stark contrast to that of developed countries; we found an unreasonable number housekeeping staff (22\%) suffering from SIs. ${ }^{6}$ The earlier data was also from the same authors and institutes as that of current publication. Probably this was due to inappropriate biomedical waste disposal practice, which is widely prevalent in India. Our observation was supported by yet another Indian study which showed 33\% of injuries among 'attendants'. After publication of our work, ${ }^{6}$ based on the observations therein, the participating hospitals made efforts to introduce changes to improve upon issues which needed change and continued to collect data using the EPINet format. This report is the analysis of prospective EPINet data from five hospitals from India over a period of 144 cumulative months and 1518321 IP days. The results of this data would show not only the prevailing practices among these hospitals, but also results of efforts of the members of the participating hospitals towards steps taken to reduce SIs among HCWs.

\section{Methods}

Five Indian hospitals (A, B, C, D, and E) participated in data collection using the EPINet data collection software. The authors of the earlier publication are the authors of this work also. All the five hospitals that participated in the earlier work also participated in this study. The first set of data for the period of June 2008 through May 2009 was collected ("data A"), analysed and published. $^{6}$ After this publication, certain changes were brought about by each participating member's healthcare facility to address the problems that were identified in that study. The data from June 2009 through June 2011 from all the five participating members was collated and this data (Data B) probably reflects the effects of those changes. The epidemiological aspects of the data in toto (termed "Total Data") was also analysed. All the participating members used the software EPINet described by Jagger, from the University of Virginia, United States of America (8). The hospitals were also requested to reply to a set of questions after initial analysis of data and the responses from participants were also analysed.
Definitions of SI, needle stick prevention safety device, a necessary/unnecessary needle or sharp device, potentially preventable SIs were the same as in our earlier publication. ${ }^{6}$ The data collection forms were supplied by the International Health Care Worker Safety Council, University of Virginia The variables analysed were as follows: Job categories, location at the hospital where the exposure occurred, whether the object causinginjury was infected, whether the source of the contaminated item was known, whether the injured worker the original user, type of the device causing injury, whether a safety device was used, the location on the corpus of the HCW and the depth of the injury, and the vaccination status of the HCW.

\section{Results}

The incidence of SIs during the two time periods and the total SI were as follows: six hundred sixty six SIs were noted in more than 1.5 million IP days (0.44/1000 IP days) and the cumulated one hundred forty four months of data collection starting June 2008 (0.43/1000 IP days). Data A contained one hundred sixty nine injuries over cumulated 39 months and about 70000 IP days (0.24/1000 IP days) and data B, contained 497 injuries during the cumulated 105 months and about 82000 IP days (0.60/1000 IP days). Two hundred thirty one nurses suffered SIs in the first period (0.01 SIs/1000 IP days) in contrast to 327 during the second (0.02/1000 IP days). Hospital A showed a threefold increase from the first period to the second in the injury to the nurses from 29 to 89 and hospital C from 9 to 32. The injury among the nontechnical staff involved in transport and disposal of biomedical waste increased from $0.004 / 1000$ IP days to 0.01 and in the technicians in the laboratory and blood bank it increased from 0.04 to $0.01 / 1000$ IP days. Hospital A contributed to most of the 'others' category with 32 injuries out of the total 43.

Table 1 shows the percentage of injury within the group. The injury among the nurses nearly doubled from 0.01 in data A to 0.02 in data B, but overall, fewer nurses were injured (46.5 v/s 56.8\%). The injury pattern among doctors and 'others' remained the same while a $7 \%$ increase in the injury to the housekeeping (helpers, or attendants or cleaners) staff was noted. The overall SIs rate among doctors was 0.005/1000 IP days, when data A (0.02) was compared with data B (0.01) it showed a declining trend. The incidence of SIs among housekeeping personnel increased from 0.004 in data A to 0.01 in data $\mathrm{B}(17-25 \%)$.

Table 2 shows the locations at which the SIs occurred. The overall incidence similar to data A. however, certain locations such as operation theatre and the utility area witnessed 
Table 1 - Sharp injuries as per job categories $-\boldsymbol{n}(\%)$.

\begin{tabular}{|c|c|c|c|c|c|c|}
\hline \multirow[t]{2}{*}{ Job Categories } & \multicolumn{2}{|c|}{ Data A } & \multicolumn{2}{|c|}{ Data B } & \multicolumn{2}{|c|}{ Total data } \\
\hline & $n(\%)$ & SI/1000 IP days & $n(\%)$ & SI/1000 IP days & $n(\%)$ & SI/1000 IP days \\
\hline Doctors & $15(8.9 \%)$ & 0.02 & $57(11.5 \%)$ & 0.001 & $87(10.8 \%)$ & 0.005 \\
\hline Nurses & 96 (56.8\%) & 0.01 & 231 (46.5\%) & 0.02 & 327 (49.1\%) & 0.02 \\
\hline Housekeeper & $30(17.8)$ & 0.0042 & 125 (25.2\%) & 0.01 & 155 (23.1\%) & 0.01 \\
\hline Others & $28(16.6 \%)$ & 0.004 & $84(16.9 \%)$ & 0.01 & $97(16.8 \%)$ & 0.006 \\
\hline IP days & 698029 & & 820292 & & 1518321 & \\
\hline Total & 169 & & 482 & & 666 & \\
\hline
\end{tabular}

Data A: the data from June 2008 through May 2009.

Data B: the data from June 2009 through June 2011.

SI: Sharp injuries; IP: Inpatient.

increased incidence compared to data A. Locations such as outside the patient room, emergency room, dialysis room, procedure room and phlebotomy room witnessed decreased in the SI rate.

The data revealed that more source patients were identifiable currently in contrast to data A, from $62.5 \%$ to 70.6 . However, there was an increase in the 'source unknown' from 12.9 to $15.1 \%$ and a decrease in NA category (uncontaminated sharp injury) from 2.4 to $0.2 \%$. The details whether the injured worker was the user was analysed and the overall incidence of user getting SIs was about $50 \%$. The incidence of nonuser getting SIs increased by $9 \%$ in data $\mathrm{B}$ in contrast to data $\mathrm{A}$; there were no unknown/NA persons in the list of injured HCWs.

Table 3 shows the details about the type of device causing SIs. Data A showed high incidence of injury caused by the hypodermic needle on a disposable syringe. In contrast, data B shows $5 \%$ reduction in such injuries but a fourfold increase in the SIs caused by hypodermic needle unattached to syringe. This surge was observed only among the 2 of the five participating hospitals. The injuries caused by winged steel needle (scalp vein needle) was not observed in the recent series of data. A $10 \%$ increase in contaminated SIs while a similar observation was made in the 'unknown' category.

\begin{tabular}{llll}
$\begin{array}{l}\text { Table 2 - Location in hospital where injuries occured }-\boldsymbol{n} \\
\text { (SIs/1000 IP days). }\end{array}$ & Data A & Data B & Total \\
\hline Location & 169 & 497 & 666 \\
\hline$n$ & 698,029 & 820,292 & $1,518,321$ \\
IP days & $63(0.9)$ & $183(0.22)$ & $246(0.16)$ \\
Patient room/ward & $21(0.03)$ & $37(0.04)$ & $58(0.03)$ \\
Outside patient room & $18(0.03)$ & $57(0.06)$ & $75(0.05)$ \\
ICU & $24(0.03)$ & $85(0.1)$ & $109(0.07)$ \\
OR/recovery room & $11(0.01)$ & $22(0.02)$ & $33(0.02)$ \\
ER/OPD & $3(0.003)$ & $8(0.006)$ & $11(0.006)$ \\
Lab/blood bank & $10(0.01)$ & $17(0.02)$ & $27(0.02)$ \\
PR/DF/VC & $5(0.005)$ & $59(0.04)$ & $64(0.04)$ \\
Others/NA & $4(0.005)$ & $29(0.02)$ & $33(0.02)$ \\
Service/utility area &
\end{tabular}

Data A: the data from June 2008 through May 2009.

Data B: the data from June 2009 through June 2011.

IP: Inpatient; ICU: Intensive care unit; ER: Emergency room; OPD: Outpatient department; Lab: Laboratory; PR: Procedure rooms; DF: Dialysis facility; VC: Venepuncture center.
Contaminated non-needle SIs was not observed in data B. In the next table (Table 4), the timing of injury is shown and one can observe a decrease in the injury rate during the use of the items while an increase 'during recapping of the needles' and 'after disposal, item protruding from trash bag'. The incidence 'during steps of a multi-step procedure and dissembling of equipment' did not change in comparison to Data A.

Data about the purpose of use of devices is shown in Table 5. The distribution of injuries among the unknown/not applicable, and others contributed to the majority of the injuries. The injuries caused during intramuscular/intravenous injection, connection of IV line and sampling of blood were non-avoidable ones; they contributed to about $30 \%$ of the injuries. The use of safety engineered devices (SED) was lesser (Table 6) in data B (4\%) in contrast to data A (29.8\%). There was no change in the incidence of injury to the hand between data A data B. The total data showed a total of 813 injuries, of which 597 were to the hands, of which 155 were superficial, 311 were moderately deep and 22 were deep with profuse bleeding. In data A we observed that only $50 \%$ of the HCWs were vaccinated, while in data $B$, the majority were vaccinated with hepatitis $B$ vaccination. $61 \%$ had received all the three doses, while $22 \%$ had received either 1 or 2 doses and only 13.7 had none.

\section{Discussion}

This is a prospective multi-centric study conducted at five major Indian hospitals. The infrastructural details of the

Table 3 - Type of devices causing injuries $-\boldsymbol{n}(\%)$.

\begin{tabular}{llll} 
& Data A & Data B & Total data \\
\hline Inpatient days & 698,029 & 820,292 & 1,518321 \\
Needle on disposable syringe & $60(42)$ & $155(35.8)$ & $215(37.3)$ \\
Intravenous catheter & $15(10.8)$ & $38(8.8)$ & $53(9.2)$ \\
$\quad$ & & & \\
$\quad$ stylet/needle & $9(6.2)$ & $30(6.9)$ & $39(6.7)$ \\
Suture needle & $14(9.8)$ & $144(33.2)$ & $158(27.4)$ \\
Needle, unattached hypodermic & $17(11.8)$ & 0 & $17(3)$ \\
Needle, winged steel & $28(19.5)$ & $66(15.2)$ & $94(16.3)$ \\
Others & &
\end{tabular}

Data A: the data from June 2008 through May 2009.

Data B: the data from June 2009 through June 2011. 
Table 4 - Time period when injury occurred $-\mathbf{n}(\%)$

\begin{tabular}{|c|c|c|c|}
\hline & Data A & Data B & $\begin{array}{l}\text { Total } \\
\text { data }\end{array}$ \\
\hline During use of item & $46(28.2)$ & $96(20.2)$ & $142(22.3)$ \\
\hline $\begin{array}{l}\text { Between steps of a multi-step } \\
\text { procedure }\end{array}$ & $13(8)$ & $47(10)$ & $60(9.4)$ \\
\hline Disassembling device or equipment & $12(7.3)$ & $15(3.1)$ & $27(4.2)$ \\
\hline While recapping a used needle & $15(9.2)$ & $58(12.2)$ & $73(11.4)$ \\
\hline Other after use, before disposal & $34(20.8)$ & $92(19.4)$ & $126(19.8)$ \\
\hline $\begin{array}{l}\text { Device left on floor, table, bed or } \\
\text { other inappropriate }\end{array}$ & $11(6.7)$ & $37(7.1)$ & $48(7.5)$ \\
\hline $\begin{array}{l}\text { After disposal, item protruding } \\
\text { from trash bag }\end{array}$ & $6(3.6)$ & $45(9.5)$ & $51(8)$ \\
\hline Others & $26(16)$ & $83(17.5)$ & 109 (17.1) \\
\hline
\end{tabular}

Data A: the data from June 2008 through May 2009.

Data B: the data from June 2009 through June 2011.

hospitals are the same as mentioned earlier in our publication. ${ }^{6}$ Data A and data B were compared. The incidence of various issues specified by the EPINet software were compared. Data on injuries among HCWs in India are sparse. ${ }^{7}$ From the available ones, ${ }^{8-12}$ it was observed that nurses figured prominently. This prospective data showed that the total number of SIs/1000 IP days in the 'total data' was 0.43 . The rates in data $A$ and $B$ were 0.24 and 0.60 respectively, showing a two and half fold increase in the SIs rate per 1000 IP days. The reasons for such increase are not obvious; however, we hypothesize that improvement in reporting pattern, actual increase in the rate of SIs and failure of mechanisms instituted to reduce SIs are the possible reasons. Long working hours, extended shifts and lack of sleep have been implicated in increasing the SIs. ${ }^{13-15}$ The hours of work and sleep pattern

Table 5 - Purpose of the sharp item $-\mathbf{n}(\%)$.

\begin{tabular}{lccc} 
& Data A & Data B & $\begin{array}{c}\text { Total } \\
\text { data }\end{array}$ \\
\hline Unknown/not applicable & $27(16)$ & $122(24.9)$ & $149(22.6)$ \\
Injection, intramuscular/ & $47(27.8)$ & $70(14.3)$ & $117(17.8)$ \\
$\quad$ subcutaneous (syringe) & & & \\
To connect IV line & $8(4.7)$ & $22(4.5)$ & $30(4.6)$ \\
Other injection into IV Site or port & $3(1.8)$ & $6(1.2)$ & $9(1.4)$ \\
To start intravenous infusion (IV) & $1(0.6)$ & $10(2)$ & $11(1.7)$ \\
To draw a venous blood & $13(7.7)$ & $47(9.6)$ & $60(9.1)$ \\
Obtain a body fluid or tissue sample & $2(1.2)$ & $22(4.5)$ & $24(3.6)$ \\
To draw arterial blood & $4(2.4)$ & $7(1.4)$ & $11(1.7)$ \\
Cannulate arterial or central line & $4(2.4)$ & $7(1.4)$ & $11(1.7)$ \\
Fingerstick/heelstick & $6(3.6)$ & $20(4.1)$ & $26(3.9)$ \\
Suturing & $12(7.1)$ & $33(6.7)$ & $45(6.8)$ \\
Cutting & $6(3.6)$ & $11(2.2)$ & $17(2.6)$ \\
To contain a specimen & $1(0.6)$ & $1(0.2)$ & $2(0.3)$ \\
Electrocautery & $2(1.2)$ & 0 & $2(0.3)$ \\
Cannulate IV or set up heparin lock & $1(0.6)$ & $6(1.2)$ & $7(1.1)$ \\
Flushing & $2(1.2)$ & $2(0.4)$ & $4(0.6)$ \\
Others & $22(13)$ & $83(16.9)$ & $105(15.9)$ \\
N-A & $8(4.7)$ & $27(5.5)$ & $35(5.3)$ \\
\hline Data A: the data from June 2008 through May 2009. & \\
Data B: the data from June 2009 through June 2011. & \\
NA - not applicable. & \multicolumn{4}{l}{} \\
\hline
\end{tabular}

Table 6 - Safety design available.

\begin{tabular}{lccc} 
& Data A & Data B & Total data \\
\hline Safety design (SEMD) & $34(20.1)$ & $19(3.9)$ & $53(8)$ \\
Not a safety design & $114(67.5)$ & $467(95.3)$ & $581(88.2)$ \\
Unknown & $11(6.5)$ & $4(0.8)$ & $15(2.3)$ \\
NA & $10(5.9)$ & 0 & $10(1.5)$ \\
\hline
\end{tabular}

Data A: the data from June 2008 through May 2009.

Data B: the data from June 2009 through June 2011.

SEMD: Safety engineered medical device; NA - not applicable.

on the night before the injuries were not captured in this study. All the participating hospitals confirmed in the response to the questionnaire that they conducted repeated training sessions to their HCWs aiming to improve the awareness and the importance of reporting SIs.

Noting decreasing trend of SIs among doctors and nurses (Table 1) is heartening. From amongst the few publications about this aspect from India, it is not uncommon to see nurses, nursing students and nursing interns suffering the majority of the SIs. ${ }^{7,9,11,16}$ The results of our work concurs with these observations. It is commonly opined by most of the workers that education, training, providing suitable sharp disposal units at bedside and measures towards prevention of SIs go a long way in reducing the incidence of SIs among nurses. The decrease in the incidence of SIs among nurses is probably due to the training, education and infrastructural support provided by the healthcare facilities. A disturbing observation is surge in the incidence of SIs among the housekeeping personnel from 0.004 during 2009 to 0.01 SIs/ 1000 IP days. Injury among such staff is directly as a result of improper waste disposal. Jayant and co-workers reported that $26 \%$ of their housekeeping staff had SIs, similar to our earlier observation. ${ }^{16}$ High incidence of SIs (64\%) among housekeeping is reported from Turkey. ${ }^{17}$

The commonest location of sharp injury was in the patient's room (Table 2). The number of injuries in the patient's room decreased from 0.9/1000 IP days in data A to 0.2 in data B. The remarkable decrease may have been due to providing sharp disposal units every bedside. At the author's unit, sharp disposal container was provided in the injection trolley. Injection trolley is a trolley consisting of all the equipment and medications required for carrying out injections. After analysis of data A, this change was instituted, which apparently brought about a decrease in the SIs in the patient's room. It appears that it is common to observe high incidence of SIs in the patient's room even in other countries. ${ }^{18,19}$ The next common location of injury was the intensive care unit. This location also appears to be common in most intensive care units globally. ${ }^{18,19}$ All the participating hospitals confirmed that every one of them made efforts in their facilities to reduce the SIs in the patient's room by one or more of the following measures: Providing puncture proof sharp container on the injection tray, use of prosafe vacutainer (BD India), training to prevent recapping of needles and reusing of used sharps. These measures seemed to have made the palpable impact which was observed.

The source of the sharp was identifiable in about $65 \%$ of the times. There was a slight increase in the number of 
instances when the source was not known (13\% in data A to $16 \%$ in data B). As mentioned earlier, the unknown sources are those caused by improper sharp disposal; this fact explains the increase in the instances of housekeeping staff getting SIs. Episodes of housekeeping staff getting SIs is absolutely preventable. A change in the attitude of the HCWs is all that is required. It is said that it is easier to change the equipment than the attitude of the HCW. Use of SEDs would not only decrease the SIs in the patient's rooms but also the ones that housekeeping staff suffer during transport of biomedical waste. Jagger and co-workers reported the effectiveness of safety engineered devices in reducing sharps injuries, and the importance of national-level regulations (accompanied by active enforcement) in ensuring wide-scale availability and implementation of protective devices to decrease healthcare worker risk. ${ }^{20}$ Similar outcomes were shown by Azar-Cavanagh. ${ }^{21}$ Though there is a general agreement on reduction of SIs after routine use of SEDs, a few controversies exist. ${ }^{22,23}$ It is also postulated that reporting of SIs during the use of SED would be more rigorous, because the occurrence was against the healthcare worker's expectation. Therefore, there may be a bias in reporting higher SIs when SEDs are used.

There was an increase in the number of sources patient identified from $62.5 \%$ to $70.6 \%$, and a slight increase in the incidence of users of sharps getting SIs in data B in contrast to data A. This suggests that doctors (most common users of sharps) need further education and training. The compliance of this group of HCW is poor to education and training methods; that is the biggest challenge in instituting programs to prevent SIs. ${ }^{24}$ Recommendations from some of the workers about switching over to blunt needles, use of diathermy instead of knife does not seem to have found many takers among the surgeons. ${ }^{25,26}$

The 'finger print' of the type of device causing injury (Table 3) remains more or less similar between data $\mathrm{A}$ and data B. It is not completely unexpected to see less injuries among HCWs due to winged steel needle; this device used very sparingly nowadays. Only in two of the five participating hospitals, 'hypodermic needles unattached to syringes' caused a significant number of SIs. Apparently, in those hospitals, hypodermic needles were used to 'finger prick' during blood sugar testing. It is hoped that with the revelation about it now, this practice would soon stop.

There was an increase in the number of SIs with contaminated sharps. It was pointed out that improper handling of sharps by housekeeping staff might be one of the reasons of such an increase. Three of the participating hospitals observed that rapid attrition and turnover of the housekeeping staff was the main reason for their ignorance; expected degree of training at induction was not possible. In India most hospitals, outsource these resources, which may be one of the reasons for increasing trend of SIs among housekeeping workers. Table 4 shows the timing of the injury. It is deplorable to find that the findings of data B is in no way better than data A. Only two steps at which SIs declined were 'dissembling device and equipment' and 'after use, before disposal'. A general thumb rule that was followed in the participating hospitals was that the person responsible for using the sharp would dispose them. This suggestion may have brought down the rate 'after use, before disposal', which earlier used to be carried out by yet another HCW, thus exposing him/her to SIs.

The detail about the purpose for which the sharp was used is shown in Table 5. There was a remarkable decrease (from 27.8 to $14.3 \%$ ) in the SIs caused by hypodermic needles used for intramuscular or intravenous injections. This improvement may have occurred due to tremendous efforts by all the participating hospitals towards avoiding recapping and disposing sharps in the patients' room itself, instead of carrying them over to a central point of disposal. Table 6 shows if SEDs were used. In the data A about 33\% of the devices were safety engineered, but in data B, it was only $4.7 \%$. Although it has been convincingly shown by many authors that SEDs help decrease SIs, the acceptance in India is not very forthcoming. ${ }^{20-22,27}$ It is indeed heartening to note decrease in SIs rates despite not using SEDs. It is a moot point about the extent to which SIs could be reduced had SEDs were used. It is hard to understand the difficulty in implementation of the use of SEDs in Indian hospital. The increased cost that one is required to spend in order to use SEDs might play a major factor in creating resistance to use among physicians. It is the duty of healthcare worker safety related organizations and the manufacturers of SEDs to impact the end users and hospital administrators on the cost effectiveness of SEDs. In a large multi-centric French study, Tosini and co-workers showed decreased rates of SIs, they wondered if the increased cost incurred by using SEDs was worth. They recommended that further studies be conducted to get clarity about this point. ${ }^{28}$ By implementing SEDs in their hospital, Valls and coworkers showed that the direct cost increase was 0.558 euros per patient in the emergency department and 0.636 euros per patient-day in the hospital wards. ${ }^{29}$ Glenngård and Persson showed that use of SEDs was cost effective with an offset of about 6.7 million Euros from 18 Swedish hospitals by using SEDs. ${ }^{30}$

Potential weaknesses of our study: The hospitals involved in the study are all corporate hospitals following very high standards of healthcare. The results obtained from these hospitals may not reflect the actual prevailing infection control practice of our country. Multi-centric studies from India including all tiers of hospitals may do so; such studies are the priority.

\section{Conclusions}

With the introduction of a few changes in response to the observations made from our earlier data seems to have benefited our current practice and incidence of SIs. Avoidable SIs such as to the housekeeping staff has not been achieved; this is due to improper sharp disposal. Use of SEDs is not popular in India.

\section{Conflicts of interest}

All authors have none to declare. 


\section{R E F E R E N C E S}

1. Yoshikawa T, Kidouchi K, Kimura S, Okubo T, Perry J, Jagger J. Needle stick injuries to the feet of Japanese healthcare workers: a culture-specific exposure risk. Infect Control Hosp Epidemiol. 2007;28:215-218.

2. Bi Peng, Tully PJ, Boss K, Hiller JE. Sharps injury and body fluid exposure among health care workers in an Australian tertiary hospital. Asia Pac J Public Health. 2008;20:139-147.

3. Adegboye AA, Moss GB, Soyinka F, Kreiss JK. The epidemiology of needle stick and sharp instrument accidents in a Nigerian hospital. Infect Control Hosp Epidemiol. 1994;15:27-31.

4. Azap A, Ergönül O, Memikoğlu KO, et al. Occupational exposure to blood and body fluids among health care workers in Ankara, Turkey. Am J Infect Control. 2005;33:48-52.

5. Venier AG, Vincent A, L'heriteau F, et al. Surveillance of occupational blood and body fluid exposures among French healthcare workers in 2004. Infect Control Hosp Epidemiol. 2007;28:1196-1201.

6. Chakravarthy M, Singh S, Arora A, Sengupta S, Munshi N. The epinet data of four Indian hospitals on incidence of exposure of healthcare workers to blood and body fluid: a multicentric prospective analysis. Indian J Med Sci. 2010;64:540-548.

7. Mehta A, Rodrigues C, Ghag S, Bavi P, Shenai S, Dastur F. Needlestick injuries in a tertiary care centre in Mumbai, India. J Hosp Infect. 2005;60:368-373.

8. Jagger J, Perry J. Power in numbers: using EPINet data to promote protective policies for healthcare workers. J Infus Nurs. 2002;25:S15-S20.

9. Guruprasad Y, Chauhan DS. Knowledge, attitude and practice regarding risk of HIV infection through accidental needlestick injuries among dental students of Raichur, India. Natl J Maxillofac Surg. 2011;2:152-155.

10. Salelkar S, Motghare DD, Kulkarni MS, Vaz FS. Study of needle stick injuries among health care workers at a tertiary care hospital. Indian J Public Health. 2010;54:18-20.

11. Muralidhar S, Singh PK, Jain RK, Malhotra M, Bala M. Needle stick injuries among health care workers in a tertiary care hospital of India. Indian J Med Res. 2010;131:405-410.

12. Mehta A, Rodrigues C, Singhal T, et al. Interventions to reduce needle stick injuries at a tertiary care centre. Indian J Med Microbiol. 2010;28:17-20.

13. Fisman DN, Harris AD, Rubin M, Sorock GS, Mittleman MA. Fatigue increases the risk of injury from sharp devices in medical trainees: results from a case-crossover study. Infect Control Hosp Epidemiol. 2007;28:10-17.

14. Ilhan MN, Durukan E, Aras E, Türkçüoğlu S, Aygün R. Long working hours increase the risk of sharp and needlestick injury in nurses: the need for new policy implication. J Adv Nurs. 2006;56:563-568.
15. Trinkoff AM, Le R, Geiger-Brown J, Lipscomb J. Work schedule, needle use, and needlestick injuries among registered nurses. Infect Control Hosp Epidemiol. 2007;28:156-164.

16. Jayanth ST, Kirupakaran H, Brahmadathan KN, Gnanaraj L, Kang G. Needle stick injuries in a tertiary care hospital. Indian J Med Microbiol. 2009;27:44-47.

17. Toraman AR, Battal F, Ozturk K, Akcin B. Sharps injury prevention for hospital workers. Int J Occup Saf Ergon. 2011;17:455-461.

18. Watterson L. Monitoring sharps injuries: EPINet surveillance results. Nurs Stand. 2004;5(19):33-38.

19. Wang FD, Chen YY, Liu CY. Analysis of sharp-edged medicalobject injuries at a medical center in Taiwan. Infect Control Hosp Epidemiol. 2000;21:656-658.

20. Jagger J, Perry J, Gomaa A, Phillips EK. The impact of U.S. policies to protect healthcare workers from bloodborne pathogens: the critical role of safety-engineered devices. J Infect Public Health. 2008;1:62-71.

21. Azar-Cavanagh M, Burdt P, Green-McKenzie J. Effect of the introduction of an engineered sharps injury prevention device on the percutaneous injury rate in healthcare workers. Infect Control Hosp Epidemiol. 2007;28:165-170.

22. Orenstein R, Reynolds L, Karabaic M, Lamb A, Markowitz SM, Wong ES. Do protective devices prevent needlestick injuries among health care workers? Am J Infect Control. 1995;23:344-351.

23. Black L, Parker G, Jagger J. Chinks in the armor: activation patterns of hollow-bore safety-engineered sharp devices. Infect Control Hosp Epidemiol. 2012;33:842-844.

24. Stein AD, Makarawo TP, Ahmad MF. A survey of doctors' and nurses' knowledge, attitudes and compliance with infection control guidelines in Birmingham teaching hospitals. J Hosp Infect. 2003;54:68-73.

25. Dauleh MI, Irving AD, Townell NH. Needle prick injury to the surgeon-do we need sharp needles? J R Coll Surg Edinb. 1994;39:310-311.

26. Raahave D, Bremmelgaard A. New operative technique to reduce surgeons' risk of HIV infection. J Hosp Infect. 1991;18(suppl A):177-183.

27. Sohn S, Eagan J, Sepkowitz KA, Zuccotti G. Effect of implementing safety-engineered devices on percutaneous injury epidemiology. Infect Control Hosp Epidemiol. 2004;25:536-542.

28. Tosini W, Ciotti C, Goyer F, et al. Needlestick injury rates according to different types of safety-engineered devices: results of a French multicenter study. Infect Control Hosp Epidemiol. 2010;31:402-407.

29. Valls V, Lozano MS, Yánez R, et al. Use of safety devices and the prevention of percutaneous injuries among healthcare workers. Infect Control Hosp Epidemiol. 2007;28:1352-1360.

30. Glenngård AH, Persson U. Costs associated with sharps injuries in the Swedish health care setting and potential cost savings from needle-stick prevention devices with needle and syringe. Scand J Infect Dis. 2009;41:296-302. 